\title{
Evaluation of Efficacy of Injectable-guided Tissue Regeneration with and without Clindamycin on the Colonization of Porphyromonas gingivalis by Real-time Polymerase Chain Reaction
}

\author{
Voruganti Deepak ${ }^{1}$, Johnson J Josephin ${ }^{2}$, Palaparthy R Babu ${ }^{3}$, Vikram R Guntakandla4, Jagadish R Gooty ${ }^{5}$,
} Suryakanth Malgikar ${ }^{6}$, Abhinav Gupta ${ }^{7}$

\begin{abstract}
Aim: Aim of the present study was to evaluate the loading of clindamycin with injectable-guided tissue regeneration (GTR) will prevent the colonization of Porphyromonas gingivalis and to compare and assess the quantitative changes in P. gingivalis colony forming units (CFUs) by real-time polymerase chain reaction (PCR) analysis.

Materials and methods: Thirty microbiological samples were pooled from the deepest periodontal pockets from the thirty sites from the two groups: group I-injectable GTR placed in the defect filled with demineralized freeze-dried bone allograft (DFDBA) and group II—clindamycin loaded injectable GTR placed in the defect filled with DFDBA. The total number of $P$. gingivalis CFUs was estimated using real-time PCR at baseline and 4 weeks after therapy.

Results: A significant reduction in P. gingivalis CFUs at the end of 4 weeks was seen in both groups. Comparative evaluations between both groups at 4 weeks were with a mean of $4.44 \pm 2.28$ and $4.75 \pm 3.32$, respectively. Though there was a significant reduction in group II, the difference was statistically insignificant.

Conclusion: The results suggest that clindamycin is beneficial in reducing microbial infection and can potentiate regeneration through host modulation.

Clinical significance: Injectable GTR has the ability to mold according to the defect size and shape and eliminates the need to manipulate the membrane as required for the conventional membrane.

Keywords: Clindamycin, Injectable-guided tissue regeneration, Periodontitis, P. gingivalis, Real-time PCR.

The Journal of Contemporary Dental Practice (2020): 10.5005/jp-journals-10024-2742
\end{abstract}

\section{INTRODUCTION}

Chronic periodontitis is an infectious disease resulting in inflammation within the supporting tissues of teeth, progressive attachment, and bone losses, usually associated with the presence of plaque and calculus associated with a variable microbial pattern. ${ }^{1}$ The importance of bacteria in the etiology of periodontal pockets has been clearly established. ${ }^{2,3}$ Porphyromonas gingivalis plays an important role in provoking periodontal disease. Porphyromonas gingivalis impairs innate immunity in ways that alter the growth and development of the entire biofilm, triggering a destructive change in the normally homeostatic host-microbiota interplay in the periodontium. ${ }^{4}$ Periodontal regeneration follows a variety of surgical approaches and therapeutic modalities that are used for restoring the periodontal osseous defects, such as the use of organic or synthetic barrier membranes (GTR) and the use of bone grafts and bone replacement materials. ${ }^{5}$ Bioabsorbable membranes, such as polycaprolactone $(\mathrm{PCL})$ is a viable option for many applications in tissue-engineering approaches is considered as satisfactory candidate for GTR due to its useful properties such as biocompatibility, proper mechanical strength, biodegradability, and ease of fabrication..$^{6-8}$ Guided tissue regeneration in the injectable form has the advantage of better retaining ability in the defect area as it well adapts to the morphology of the defect, thus avoiding the need for the manipulation as in case of conventional membranes. In order to prevent postoperative wound infection, some investigators have administered systemic
${ }^{1-7}$ Department of Periodontics and Implantology, Kamineni Institute of Dental Sciences, Nalgonda, Telangana, India

Corresponding Author: Voruganti Deepak, Department of Periodontics and Implantology, Kamineni Institute of Dental Sciences, Nalgonda, Telangana, India, Phone: +91-9989183060, e-mail: periomds@gmail.com

How to cite this article: Deepak V, Josephin JJ, Babu PR, et al. Evaluation of Efficacy of Injectable-guided Tissue Regeneration with and without Clindamycin on the Colonization of Porphyromonas gingivalis by Realtime Polymerase Chain Reaction. J Contemp Dent Pract 2020;21(1):36-40.

Source of support: Nil

Conflict of interest: None

antibiotics to the patients during the first 2-4 weeks after membrane implantation. ${ }^{9}$ However, despite the application of systemic antibiotics, the occurrence of postoperative wound infection, and abscess formation related to implanted barrier membranes have been noticed. ${ }^{10}$ This indicates that either the drug administered is not directed against the microorganisms responsible for the wound infection or that the drug does not reach the infected site at a concentration sufficiently high to inhibit the target microorganisms. An improved effect might be obtained by a local application of a proper antibiotic providing a sufficiently high concentration at the membrane site. Another advantage would be that a much lower 
dosage than that needed for systemic treatment could be used and the general effect on the normal flora at other body sites would be reduced. Clindamycin is a lincosamide group antibiotic known to have a very favorable spectrum of activity against anaerobic infections. It demonstrated excellent activity against $P$. gingivalis and $P$. intermedia with a high killing activity. ${ }^{11}$ According to the periodontal literature, there is no formulation of injectable GTR using PCL and clindamycin is available. Hence, the study was designed to evaluate whether the loading of clindamycin will prevent the colonization of $P$. gingivalis and to compare and assess the quantitative changes in $P$. gingivalis CFU in both groups by using real-time polymerase chain reaction (PCR) analysis.

\section{Materials and Methods}

\section{Study Design}

All patients were selected from the outpatient Department of Periodontics, Kamineni Institute of Dental Sciences, Narketpally, Nalgonda. The Ethical Committee approved the trial (IEC number KIDS/IEC/2016/03). Patients between 25 years and 60 years in the study were the age group of minimum 20 permanent teeth to be present, periodontal pocket depth $\geq 5 \mathrm{~mm}$, and evidence of angular defects as determined by intraoral periapical (IOPA) radiograph. Patients with the following criteria were excluded systemically compromised patients and those on medications that may interfere with wound healing, pregnant women and lactating mothers, active periodontal treatment in the last 6 months, and smokers. The nature and purpose of the study were explained to the patients and written informed consent was obtained. The study consisted of 30 sites in chronic periodontitis subjects. After fullmouth periodontal recording was performed in a specially prepared proforma. The subjects were randomly assigned by the coin toss method into groups I and II (15 in each group) to one of the following treatment modalities. Group I: injectable GTR placed in the defect filled with DFDBA. Group II: clindamycin loaded injectable GTR (Figs $1 \mathrm{~A}$ and $\mathrm{B}$ ) placed in the defect filled with DFDBA.

\section{Radiographic Evaluation}

Radiographically, the infrabony defects were recorded from a fixed reference point (cementoenamel junction) to the base of the defect. The IOPA radiographs were standardized by long cone paralleling technique and using film holders (RINN XCP ${ }^{\mathrm{TM}}$, DENTSPLY).

\section{Preparation of In Situ Gel}

Clindamycin loaded injectable GTR gel was prepared by the Department of Pharmaceutics, Vikas College of Pharmaceuticals Sciences, Suryapet (Table 1).

\section{Microbiological Procedure}

Before surgery and 4 weeks after surgery, 30 (15 in each group) subgingival plaque samples were pooled from the deepest periodontal pockets in each patient (Fig. 1C). After removing supragingival plaque and isolating the sample sites with cotton rolls, sterile endodontic fine paper points were inserted into the depth of the pocket and placed for 30 seconds. The paper points were placed into a transport medium in Eppendorf tubes containing Tris-EDTA buffer solution (Fig. 1D). The total number of $P$. gingivalis CFU were estimated using real-time PCR at baseline and 4 weeks after surgery. The real-time PCR analyses conducted in the Maratha Mandal's NGH Institute of Dental Sciences, Belagavi, Karnataka.
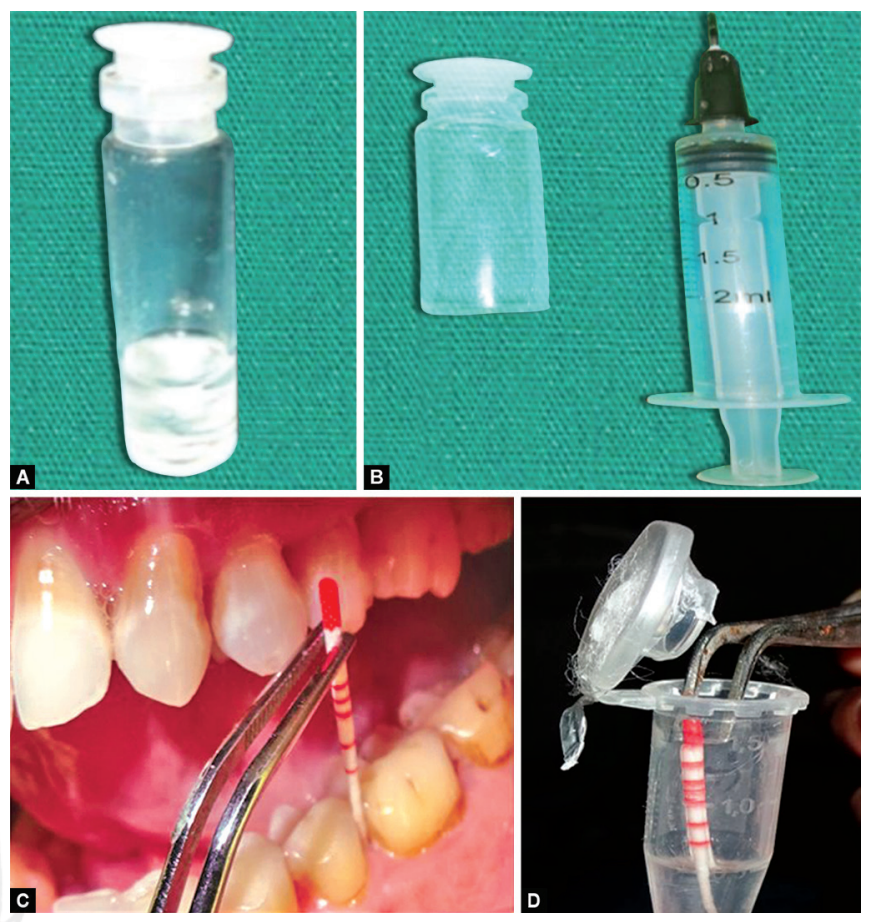

Figs $1 A$ to $D:(A)$ Clindamycin-guided tissue regeneration (GTR); (B) Injectable GTR; (C) Collection of subgingival plaque sample; (D) Paper points placed in Eppendorf tubes

Table 1: GTR formulation

\begin{tabular}{llll}
\hline \multirow{2}{*}{ Formulations } & Polymer & Drug & Solvent \\
\cline { 2 - 4 } & Polycaprolactone & Clindamycin & Dichloromethane \\
\hline F & $60 \%$ & $10 \%$ & $40 \%$ \\
\hline
\end{tabular}

\section{DNA Extraction Procedure (Modified Proteinase-K Method)}

The subgingival plaque samples were transferred to the tube containing TE buffer and centrifuged at 5,000 rpm for 5 minutes. The supernatant was discarded. About $500 \mu \mathrm{L}$ fresh T.E. buffer was added and centrifuged for 3-4 minutes (Figs $2 A$ and $B$ ). The above procedure was repeated for 3-4 times with fresh TE buffer. The supernatant was discarded, $50 \mu \mathrm{L}$ lysis buffer I was added, vortexed and kept for 5 minutes. About $50 \mu \mathrm{L}$ lysis buffer II was added and $10 \mu \mathrm{L}$ proteinase-K $(100 \mu \mathrm{g} / \mathrm{mL})$, vortexed vigorously. The mixture was stored water bath for 2 hours and then kept in a boiling water bath for 10 minutes. DNA samples were stored at $-20^{\circ} \mathrm{C}$.

\section{Real-time PCR Procedure}

During each phase of DNA synthesis, the SYBR green I dye, in the reaction mix, binds to the amplified PCR products; the amplicon can be detected by its fluorescence. Serial dilutions of the DNA extracted from the standard strain of $P$. gingivalis ATCC no. 33277 (known quantity, $10^{8}-10^{3} \mathrm{CFU} / \mathrm{mL}$ ) was also run to plot the standard graph. The SYBR green dye binds with double-stranded DNAs that were specifically amplified by $P$. gingivalis specific primers. The dye emits the fluorescence in the form of a graph. The graph was plotted as the amount of fluorescence against the number of cycles (Figs 2C and 3). Ct value (cycle no. at which fluorescence has initiated) was obtained from the graph for all the samples including standards. A standard curve was obtained from the $\mathrm{Ct}$ 

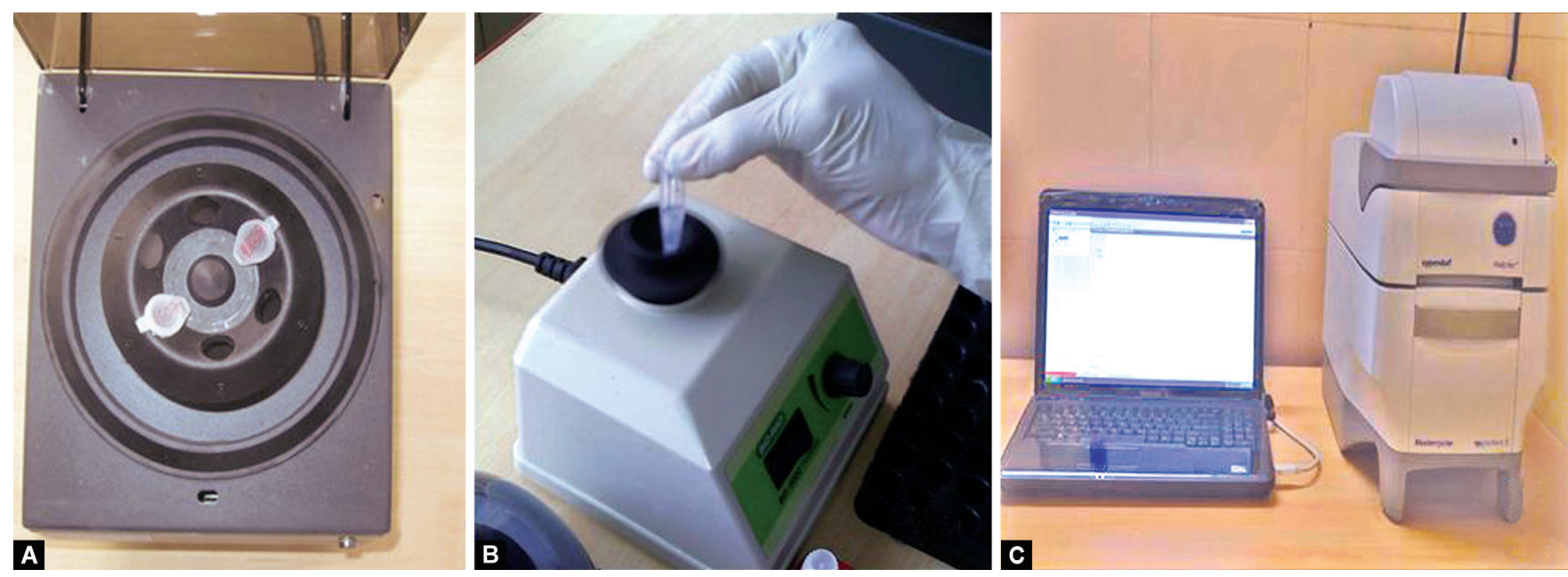

Figs 2A to C: (A) Centrifugation; (B) DNA extraction; (C) Real-time PCR analysis

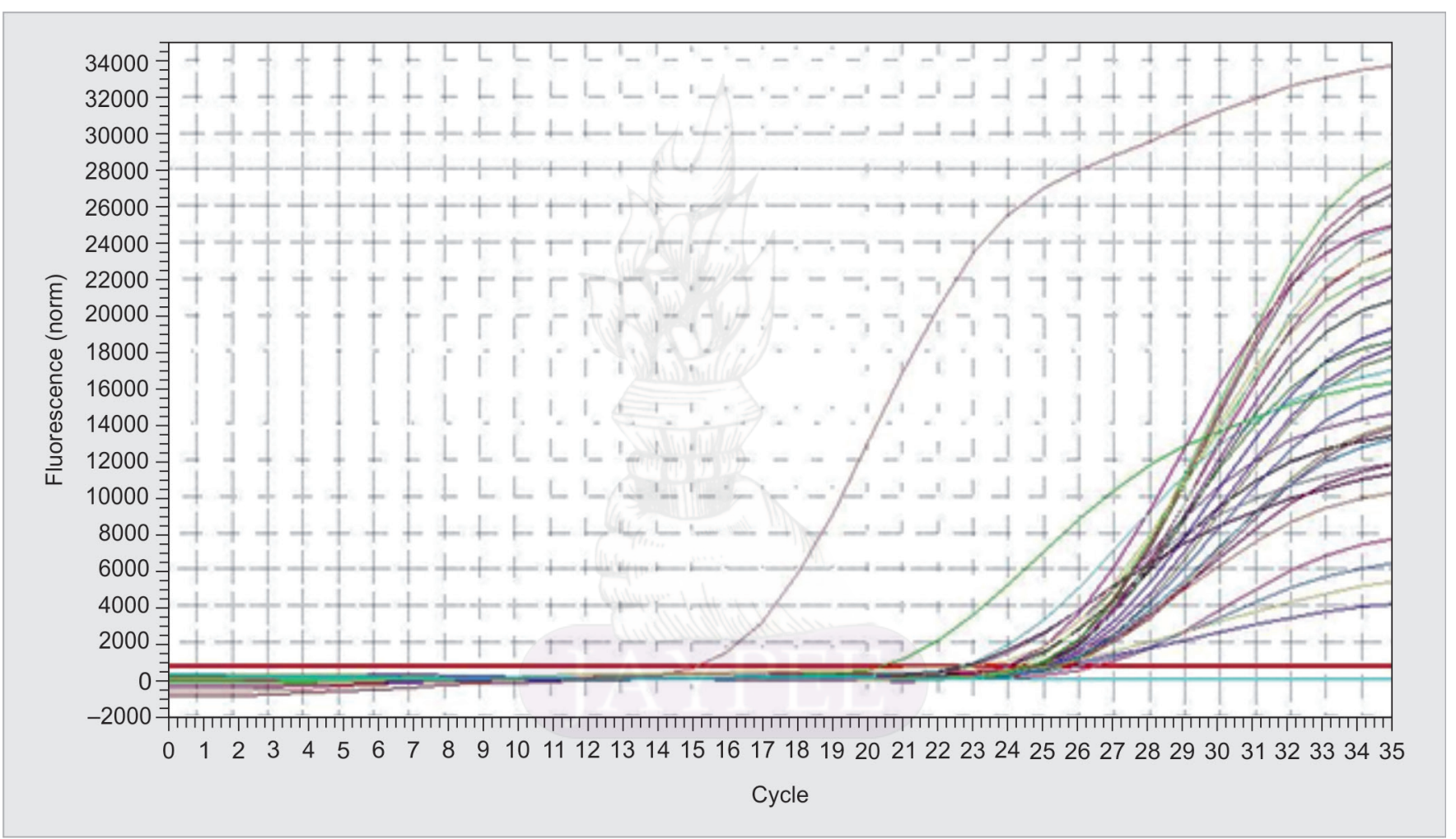

Fig. 3: Amplification plot of Porphyromonas gingivalis by real-time PCR

values of standard samples. Ct value of each sample was plotted on to the standard curve and the corresponding quantity of DNA copy numbers were obtained.

\section{Statistical Analysis}

Data were collected by using a structured proforma and analyzed by using SPSS 24.0 version IBM USA. Paired $t$ test was used to evaluate the $P$. gingivalis levels between baseline and 4 weeks and comparative evaluation between the two groups was done using the independent sample $t$ test. ${ }^{*} p<0.05$ was considered statistically significant.

\section{Results}

All the enroled patients completed the study period of 4 weeks. The $P$. gingivalis levels reduced in both group I and group II from baseline to 4 weeks. In group, I P. gingivalis levels at baseline and at 4 weeks were with a mean of $6.24 \pm 0.83$ and $4.44 \pm 2.28$, respectively (Table 2) whereas in group II $P$. gingivalis levels at baseline and at 4 weeks were with a mean of $6.67 \pm 1.06$ and $4.57 \pm 3.32$, respectively (Table 3 and Fig. 4) that were statistically insignificant between the two groups.

\section{Discussion}

Porphyromonas gingivalis is a gram-negative oral anaerobe that is involved in the pathogenesis of periodontitis, an inflammatory disease that destroys the tissues supporting the tooth, which eventually may lead to tooth loss. ${ }^{12}$ The virulence factors of $P$. gingivalis, such as lipopolysaccharide, vesicles, gingipains, and fimbriae not only directly destroy periodontal tissue but also 
Table 2: Comparison of $\log _{10}$ units of Porphyromonas gingivalis between baseline and 1 month in each study group

\begin{tabular}{|c|c|c|c|c|c|c|c|c|c|}
\hline \multirow[b]{2}{*}{ Group } & & \multirow[b]{2}{*}{$n$} & \multirow[b]{2}{*}{ Mean $\pm S D$} & \multirow[b]{2}{*}{ Mean difference } & \multicolumn{2}{|c|}{$\begin{array}{l}\text { 95\% confidence interval of the } \\
\text { difference }\end{array}$} & \multirow[b]{2}{*}{$t$} & \multirow[b]{2}{*}{$d f$} & \multirow[b]{2}{*}{$p$ value* } \\
\hline & & & & & Lower & Upper & & & \\
\hline \multirow[t]{2}{*}{$\mathrm{I}$} & Baseline & 15 & $6.24 \pm 0.83$ & 1.80 & -0.29 & 3.89 & 2.11 & 6 & 0.08 (NS) \\
\hline & 1 month & 15 & $4.44 \pm 2.28$ & & & & & & \\
\hline \multirow[t]{2}{*}{$\|$} & Baseline & 15 & $6.67 \pm 1.06$ & 2.10 & -0.53 & 4.73 & 1.95 & 6 & 0.10 (NS) \\
\hline & 1 month & 15 & $4.57 \pm 3.32$ & & & & & & \\
\hline
\end{tabular}

${ }^{*} p<0.05$ statistically significant, $p>0.05$ nonsignificant. NS, nonsignificant

Table 3: Comparison of $\log _{10}$ units of Porphyromonas gingivalis between the study groups at baseline and 1 month

\begin{tabular}{|c|c|c|c|c|c|c|c|c|c|}
\hline & \multirow[b]{2}{*}{ Group } & \multirow[b]{2}{*}{$n$} & \multirow[b]{2}{*}{ Mean $\pm S D$} & \multirow[b]{2}{*}{ Mean difference } & \multicolumn{2}{|c|}{$\begin{array}{l}\text { 95\% confidence interval of the } \\
\text { difference }\end{array}$} & \multirow[b]{2}{*}{$t$} & \multirow[b]{2}{*}{$d f$} & \multirow[b]{2}{*}{ p value* } \\
\hline & & & & & Lower & Upper & & & \\
\hline \multirow[t]{2}{*}{ Baseline } & 1 & 15 & $6.24 \pm 0.83$ & -0.42 & -1.53 & 0.69 & -0.83 & 12 & 0.42 (NS) \\
\hline & ॥ & 15 & $6.67 \pm 1.06$ & & & & & & \\
\hline \multirow[t]{2}{*}{1 month } & I & 15 & $4.44 \pm 2.28$ & -0.12 & -3.44 & 3.19 & -0.08 & 12 & 0.94 (NS) \\
\hline & $\|$ & 15 & $4.57 \pm 3.32$ & & & & & & \\
\hline
\end{tabular}

${ }^{*} p<0.05$ statistically significant, $p>0.05$ nonsignificant. NS, nonsignificant

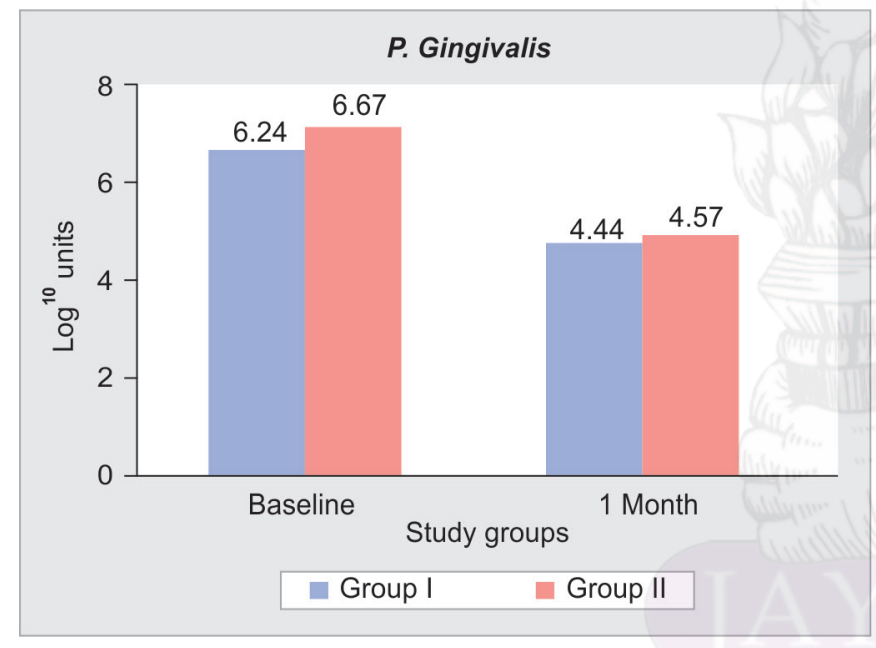

Fig. 4: Inter group comparison of two groups (I and II) with respect to Porphyromonas gingivalis levels at baseline and 1 month

cause secondary tissue damage by producing an inflammatory response. ${ }^{13}$ In other words, $P$. gingivalis could be a keystone pathogen of the disease-provoking periodontal microbiota. ${ }^{4}$ The results from systemic review and meta-analysis demonstrated that GTR technique exhibited highly variable results between and within the studies. In order to advance the healing capability of periodontal tissues, membrane modification is widely investigated. In this regard, the development of the drug/bioactive agent-containing membrane has been developed.

Polycaprolactone has been introduced as a candidate biomaterial for tissue regeneration. It has many properties that satisfy the criterion for the GTR membrane. For example, it exhibits biocompatibility properties and is not toxic. It has been widely investigated as a scaffold material for tissue-engineering application. While the GTR membrane's aim for facilitating the regeneration and healing of periodontal tissues, this type of membrane is considered as bioactive-GTR membrane. The first approach is to incorporate antimicrobial agents with the GTR membrane to attenuate the risk of bacterial infection. Among the wide variety of antimicrobials used to control infections following periodontal therapy, doxycycline has exhibited promising results. ${ }^{14}$ Antibiotic's impregnation of GTR membranes may reduce the early colonization of bacteria on the membranes. However, different in vitro conditions should be considered, as factors such as host defense mechanisms, cell type, and inter-individual fibroblast heterogeneity, and bacterial competition may be present in vivo. ${ }^{15}$ Membrane exposure is a common phenomenon in GTR treatment, which provides an environment for bacterial adherence and multiplication. ${ }^{16}$ Yoshinari et al. ${ }^{17}$ demonstrated that numerous bacteria adhered and invaded membranes accompanied by a bacterial infection.

Clindamycin reaches high concentrations in saliva, GCF, and bone. Several studies have shown that the concentration of clindamycin in these tissues is approximately $40-50 \%$ of the concentration in serum. ${ }^{18}$ In a study, the concentration of clindamycin in bone and other tissue was above the minimal concentration at which $90 \%$ of the isolates are inhibited (MIC90) for pathogens that are likely to be introduced into the tissues in patients undergoing oral and maxillofacial surgery. ${ }^{19}$ Clindamycin therapy is an effective means of treating periodontal disease due to obligate anaerobic bacilli, such as $P$. gingivalis and $P$. intermedia. The use of locally applied clindamycin gel inserted into periodontal pockets was beneficial in the treatment of advanced periodontitis by eliminating and preventing early recolonization of periodontopathogenic species and might avoid known side enhancement of the effects of systemic administration, such as antibiotic-associated pseudomembranous enterocolitis.

Considering the $\mathrm{PCL}$ as a potential agent, an attempt for the first time in the literature has been made to deliver PCL as a GTR in the gel form that can be injected into the local site. Injectable GTR has the ability to mold according to the defect size and shape and prevent the necessity to manipulate the membrane as required in the case of the conventional membrane. In this regard, clindamycin 
was added along with PCL in the form of the gel has been developed and delivered into the local site to assess the $P$. gingivalis levels. In the present study, comparative evaluations between groups I and II at 4 weeks were with a mean of $4.44 \pm 2.28$ and $4.75 \pm 3.32$, respectively. Though there was a significant reduction in group II, the difference was not statistically significant $(p=0.08)$. The results of the study partially agreed with the previous studies where they had discussed the microbiologic and clinical evidence supporting the efficacy and safety of clindamycin for the successful management of dental infections. ${ }^{20}$ This could be attributed to the inability to sustain the minimum inhibitory concentration of drug in the local site for sufficient length of time and different in vitro conditions should be considered, as factors such as host defense mechanisms, cell type, and inter-individual fibroblast heterogeneity, and bacterial competition may be present in vivo. Local administration of chlorhexidine was considered necessary but was limited to the first postoperative week when patients would most likely have difficulties with their oral hygiene. Administration of chlorhexidine could have affected bacterial postoperative colonization. The administration could have minimal but similar effects for the two groups on the microflora present within the preserved furcation space due to the limited subgingival penetration of mouth rinses.

According to the periodontal literature and the best of our knowledge, this is the first study to be conducted. Although clindamycin-loaded GTR did not show statistically significant results, from a futuristic viewpoint, attempts can be made to harness the potential additive effects of clindamycin in conjunction with periodontal surgical procedures to block the pathways of periodontal tissue destruction and to enhance wound healing and regeneration.

\section{Conclusion}

The addition of clindamycin in injectable GTR gel may lower the levels of the bacteria illustrating clindamycin as a potential drug for the application in periodontal regeneration. The other important aspect is the use of $\mathrm{PCL}$, which is a new and promising injectable GTR gel which may increase its retention after placement, unlike the conventional GTR which requires manipulation of the membrane.

\section{References}

1. American Academy of Periodontology. Glossary of periodontal terms, 4th ed. Chicago: AAP; 2001. p. 40.

2. Moore WEC. Microbiology of periodontal diseases. J Periodont Res 1987;22(5):335-341. DOI: 10.1111/j.1600-0765.1987.tb01595.x.

3. Mombelli A, Cionca A, Almaghlouth A. Does adjunctive antimicrobial therapy reduce the perceived need for periodontal surgery? Periodontol 2000 2011;55(1):205-216. DOI: 10.1111/j.16000757.2010.00356.x.

4. Hajishengallis G, Darveau RP, Curtis MA. The keystone-pathogen hypothesis. Nat Rev Microbiol 2012;10(10):717-725. DOI: 10.1038/ nrmicro2873.

5. Bansal C, Bharti V. Evaluation of efficacy of autologous plateletrich fibrin with demineralized-freeze dried bone allograft in the treatment of periodontal intrabony defects. J Indian Soc Periodontol 2013;17(3):361-366. DOI: 10.4103/0972-124X.115663.

6. FecekC, Yao D, Kacorri A, et al. Chondrogenic derivatives of embryonic stem cells seeded into 3D polycaprolactone scaffolds generated cartilage tissue in vivo. Tissue Eng Part A 2008;14(8):1403-1413. DOI: 10.1089/ten.tea.2007.0293.

7. Li WJ, Tuli R, Okafor C, et al. A three-dimensional nanofibrous scaffold for cartilage tissue engineering using human mesenchymal stem cells. Biomaterials 2005;26(6):599-609. DOI: 10.1016/ j.biomaterials.2004.03.005.

8. Li WJ, Jiang YJ, Tuan RS. Cell-nanofiber-based cartilage tissue engineering using improved cell seeding, growth factor and bioreactor technologies. Tissue Eng Part A 2008;14(5):639-648. DOI: 10.1089/tea.2007.0136.

9. Kersten BG, Chamberlain ADH, Khorsandi S, et al. Healing of the intrabony periodontal lesion following root conditioning with citric acid and wound closure including an expanded PTFE membrane. J Periodontol 1992;63(11):876-882. DOI: 10.1902/jop.1992.63. 11.876 .

10. Goodson JM, Offenbacher S, Farr DH, et al. Periodontal disease treatment by local drug delivery. J Periodontol 1985;56(5):265-272. DOI: 10.1902/jop.1985.56.5.265.

11. Gordon J, Walker C, Lamster I, et al. Efficacy of clindamycin hydrochloride in refractory priodontitis: 12 month results.J Periodontol 1985;56(Suppl 11S):75-80. DOI: 10.1902/jop.1985.56.11s.75.

12. Bodet C,Chandad F, Grenier D. Pathogenic potential of Porphyromonas gingivalis, Treponema denticola and Tannerella forsythia, the red bacterial complex associated with periodontitis. Pathol Biol (Paris) 2007;55(3-4):154-162. DOI: 10.1016/j.patbio.2006.07.045.

13. Silva $N$, Abusleme $L$, Bravo $D$, et al. Host response mechanisms in periodontal diseases. J Appl Oral Sci 2015;23(3):329-355. DOI: 10.1590/1678-775720140259.

14. Chaturvedi R, Gill AS, Sikri P. Evaluation of the regenerative potential of $25 \%$ doxycycline loaded biodegradable membrane vs biodegradable membrane alone in the treatment of human periodontal infrabony defects: a clinical and radiological study. Indian J Dent Res 2008;19(2):116-123. DOI: 10.4103/0970-9290.40465.

15. Gottlow J, Nyman S, Lindhe J, et al. New attachment formation in the human periodontium by guided tissue regeneration. Case reports. J Clin Periodontol 1986;13(6):604-616. DOI: 10.1111/j.1600-051X.1986. tb00854.x.

16. Hung $S L$, Lin $Y W$, Wang $Y H$, et al. Permeability of streptococcus mutans and Actinobacillus actinomycetemcomitans through guided tissue regeneration membranes and their effects on attachment of period. J Periodontol 2002;73(8):843-851. DOI: 10.1902/jop.2002.73. 8.843.

17. Yoshinari N, Tohya T, Mori A, et al. Inflammatory cell population and bacterial contamination of membranes used for guided tissue regenerative procedures. J Periodontol 1998;69(4):460-469. DOI: 10.1902/jop.1998.69.4.460.

18. LeFrock JL, Molavi A, Prince RA. Clindamycin. Med Clin North Am 1982;66(1):103-120. DOI: 10.1016/S0025-7125(16)31445-6.

19. Mueller SC, Henkel KO, Neumann J, et al. Perioperative antibiotic prophylaxis in maxillofacial surgery: penetration of clindamycin into various tissues. J Craniomaxillofac Surg 1999;27(3):172-176. DOI: 10.1016/S1010-5182(99)80046-8.

20. Brook I, Lewis MA, Sandor GK, et al. Clindamycin in dentistry: more than just effective prophylaxis for endocarditis? Oral Surg Oral Med Oral Pathol Oral Radiol Endod 2005;100(5):550-558. DOI: 10.1016/ j.tripleo.2005.02.086. 